BULL. AUSTRAL. MATH. SOC.

VOL. 12 (1975), 425-43I.

\title{
Oscillations of an equation relevant to an industrial problem
}

\section{Alexander Tomaras}

\begin{abstract}
The oscillatory behaviour of the solutions of a functional differential equation, of more general form than an equation arising from in industrial problem, is examined. The question of whether one can maintain the oscillatory properties of this equation by adding a forcing term is also answered. The results obtained extend already known results on the subject and complete the relevant literature.
\end{abstract}

\section{Introduction}

The problem of analysing the dynamics of an overhead current collection system for an electric locomotive has been the subject of discussion in [9], where the analysis gave rise to a system of linear first order ordinary differential equations, in which the argument of one of the dependent variables is multiplied by a factor $\lambda$. Asymptotic and numerical methods to study the properties and practical solutions of such systems were used in [4], where, in particular, a simple physical situation, which gives rise to the scalar equation

$$
y^{\prime}(t)=a y(\lambda t)+b y(t),
$$

was described.

Series and integral representations of the solutions to (1.1), where

Received 3 February 1975. The author would like to thank Dr Janet Dyson for Pruitful discussions. 
$a$ and $b$ are constants, were discussed in [4], while in [6] it was shown, that the boundary-value problem associated with (1.1) and the boundary condition $y(0)=1$, is well-posed if $\lambda<1$, but not if $\lambda>1$. Asymptotic properties of solutions of (1.1), as $t \rightarrow \infty$, were also treated in [6], as well as in [1,2 for $b=0],[3]$, and [5] and its oscillatory behaviour (only for $b=0$ ) was mentioned in [8]

The purpose of the present paper is to add information to the literature, related to ( 1.1 ), extending and generalising notions contained in it.

Thus, we examine first the oscillatory behaviour of the solutions of the functional differential equation

$$
y^{\prime}(t)=p(t) y(g(t))+q(t) y(t),
$$

which is of more general form than (1.1) - in fact, (1.1) can be derived from (1.2) for $p(t)=a, g(t)=\lambda t, q(t)=b$ - and of course, oscillatory properties concerning the solutions of (1.2), will also concern those of (1.1), under proper modifications. Secondly we extend the results obtained for (1.2) to the functional differential equation, resulting from it by adding a forcing term, namely

$$
y^{\prime}(t)=p(t) y(g(t))+q(t) y(t)+r(t) .
$$

In the sequel, a solution $y(t)$ of (1.2) or (1.3) is said to be oscizlatory, if it has arbitrarily large zeros, while it is said to be nonoscizlatory, if it is eventually of constant sign.

\section{Unforced oscillation}

In this section, we give sufficient growth conditions on $p(t), g(t)$, and $q(t)$, so that all solutions of (1.2) oscillate.

We shall need first the following lema, which is an adaptation of a corollary in $[8, \mathrm{p} .222]$.

LEMMA 2.1. Consider the functional differential equation

$$
x^{\prime}(t)+p(t) x(g(t))=0,
$$

where

(C1) $p(t) \in C\left[[0, \infty), R^{+}\right], g(t) \in C^{1}\left[[0, \infty), R^{+}\right]$, and 
(C2) $g(t)<t, \lim _{t \rightarrow \infty} g(t)=\infty$, and $g^{\prime}(t) \geq 0$.

If, in addition,

$$
\underset{t \rightarrow \infty}{\lim \sup _{g(t)}} \int_{g(s) d s>1}^{t}
$$

then every solution $x(t)$ of (2.1) oscillates.

Now, set $y(t) \exp \left(-\int q(t) d t\right)=z(t)$, to obtain (1.2) in the form

$$
z^{\prime}(t)+Z(t) z(g(t))=0,
$$

- where $Z(t)=-p(t) \exp \int_{t}^{g(t)} q(T) d T$ - which is the same as (2.1) and for which Lemma 2.1 holds of course, under minor modifications. So, observing that if $z(t)$ oscillates, so does $y(t)$, we apply Lemma 2.1 to (2.3), to establish the following result for (1.2).

THEOREM 2.1. Consider the functional differential equation (1.2) subject to the following conditions:

$$
\begin{aligned}
& \text { (i) }-p(t) \in C\left[[0, \infty), R^{+}\right] ; \\
& \text {(ii) } g(t) \in C^{l}\left[[0, \infty), R^{+}\right], g(t) \leq t, \lim _{t \rightarrow \infty} g(t)=\infty \text {, and } \\
& g^{\prime}(t) \geq 0 ; \\
& \text { (iii) } q(u) \text { is continuous for all } u \neq 0 . \\
& \text { If, in addition, }
\end{aligned}
$$

$$
\lim _{t \rightarrow \infty} \int_{g(t)}^{t}-p(\cdot s)\left[\exp \int_{s}^{g(s)} q(T) d T\right] d s>1,
$$

then every solution $y(t)$ of $(1.2)$ oscillates.

REMARKS. It would not seem too easy for someone to derive Theorem 2.1 from usual oscillation theory techniques, if he had worked directly from (1.2) (note especially condition (2.4)). This theorem, however, came out naturally, as an application of the exposition in [8], after the transformation of (1.2) to (2.3). In this fact lies the importance of that transformation, which is a new concept in the relevant literature, as far 
as we know.

On the other hand, Theorem 2.1, as a "qualitative result", completes the theory described in [6, paragraphs 4, 5, and 6] and one can now easily apply that theorem to. ( 1.1 ), under proper modifications, to extend results obtained for it, in $[8, p .222]$, for $b=0$; we leave the details to the reader.

\section{Forced oscillation}

Proceeding further, we give sufficient growth conditions on $p(t)$, $g(t), q(t)$, and $r(t)$, so that all solutions of (1.3) oscillate. To this end, in addition to the transformations established in Section 2 , set $r(t) \exp \left(-\int q(t) d t\right)=m(t)$, to obtain

$$
\boldsymbol{z}^{\prime}(t)+Z(t) \boldsymbol{z}(g(t))=m(t) .
$$

But for (3.1), the following lemma holds, which is a modification of a theorem in [7, p. 274], using Lemma 2.1 given previously.

LEMMA 3.1. Consider the functional differential equation (3.1) subject to the following hypotheses:

(hl) $Z(t), m(t) \in C[[0, \infty), R], Z(t) \geq 0$;

(h2) $g(t) \in C^{1}[[0, \infty), R], g(t) \leq t, \lim _{t \rightarrow \infty} g(t)=\infty$, $g^{\prime}(t) \geq 0 ;$

(h3) $\lim \sup _{t \rightarrow \infty} \int_{g(t)}^{t} Z(s) d s>1$;

(h4) $q(u)$ is continuous for all $u \neq 0$;

(h5) there exists a function $Q(t) \in C^{1}[[0, \infty), R]$, such that $Q^{\prime}(t)=m(t), \quad t \geq 0$, and either

(I) $\lim _{t \rightarrow \infty} Q(t)=0$, or

(2) there exist constants $q_{1}, q_{2}$ and sequences $\left\{t_{m}^{\prime}\right\},\left\{t_{m}^{\prime \prime}\right\}$, such that $\underset{m \rightarrow \infty}{\lim } t_{m}^{\prime}=\lim _{m \rightarrow \infty} t_{m}^{\prime \prime}=\infty$ and 


$$
Q\left(t_{m}^{\prime}\right)=q_{1}, \quad Q\left(t_{m}^{\prime \prime}\right)=q_{2}, \quad q_{1} \leq Q(t) \leq q_{2}, \quad t \geq 0 .
$$

Then, if (1) holds, every solution $z(t)$ of (3.1) oscillates or lim $z(t)=0$, while if (2) holds, every solution $z(t)$ of (3.1) $t \rightarrow \infty$ oscillates.

Now, using the above lemma and the previously given transformations, we establish the following result for (1.3).

THEOREM 3.1. Consider the fionetional differential equation (1.3) subject to the following hypotheses:

(HI) $p(t), r(t) \in C[[0, \infty), R], \quad-p(t) \geq 0$;

(H2) $g(t) \in C^{l}[[0, \infty), R], g(t) \leq t, \lim _{t \rightarrow \infty} g(t)=\infty$, $g^{\prime}(t) \geq 0 ;$

(H3) $\lim \sup _{t \rightarrow \infty} \int_{g(t)}^{t}-p(s) \exp \left(\int_{s}^{g(s)} q(T) d T\right) d s>1$;

(H4) $q(u)$ is continuous for alz $u \neq 0$;

(H5) there exists a function $Q(t) \in C^{\perp}[[0, \infty), R]$, such that $Q^{\prime}(t)=r(t) \exp \left(-\int q(t) d t\right), t \geq 0$ and either

(I) $\lim _{t \rightarrow \infty} Q(t)=0$, or

(II) there exist constants $q_{1}, q_{2}$ and sequences $\left\{t_{m}^{\prime}\right\},\left\{t_{m}^{\prime \prime}\right\}$, such that $\lim _{m \rightarrow \infty} t_{m}^{\prime}=\lim _{m \rightarrow \infty} t_{m}^{\prime \prime}=\infty$ and

$$
Q\left(t_{m}^{\prime}\right)=q_{1}, \quad Q\left(t_{m}^{\prime \prime}\right)=q_{2}, \quad q_{1} \leq Q(t) \leq q_{2}, \quad t \geq 0 .
$$

Then, if (I) holds, every solution $y(t)$ of (1.3) oscillates or Iim $y(t)=0$, while if (II) holds, every solution $y(t)$ of (I.3) $t \rightarrow \infty$ oscillates.

The above result accomplishes the purpose of this paper.

A QUESTION. We have seen so far, that one can deal directly with the 
oscillatory character of the solutions of (1.2) and (1.3), by transforming them properly into equations which have already been examined. Could this procedure - under suitable transformations - be applied to the higher-order retarded differential equations

$$
y^{(n)}(t)=p(t) y(g(t))+q(t) y(t)
$$

and

$$
y^{(n)}(t)=p(t) y(g(t))+q(t) y(t)+r(t),
$$

respectively, for $n>1$ ? This question remains open.

\section{References}

[1] N.G, de Bruijn, "The difference-differential equation

$F^{\prime}(x)=e^{\alpha x+\beta} F(x-1) \cdot I^{\prime \prime}, K$. Nederl. Akad. Wetensch. Proc. Ser. A 56 = Indag. Math. 15 (1953), 449-458.

[2] N.G, de Bruijn, "The difference-differential equation

$F^{\prime}(x)=e^{\alpha x+\beta} F(x-1)$. II", K. Nederl. Akad. Wetensch. Proc. Ser, A 56 = Indag. Math. 15 (1953), 459-464.

[3] Janet Dyson, "Some topics in functional differential equations", ( $D$. Phil. Thesis, Oxford, 1973).

[4] L. Fox, D.F. Mayers, J.R. Ockendon and A.B. Tayler, "On a functional differential equation", J. Inst. Math. Appl. 8 (1971), 271-307.

[5] Tosio Kato, "Asymptotic behaviour of solutions of the functional differential equation $y^{\prime}(x)=a y(\lambda x)+b y(x)$ ", Delay and functional differential equations and their applications, 197-217 (Proc. Conf. Park City, Utah, 1972. Academic Press, New York, London, 1972).

[6] Tosio Kato and J.B. McLeod, "The functional-differential equation $y^{\prime}(x)=a y(\lambda x)+b y(x)$ ", Bull. Amer. Math. Soc. 77 (1971), $891-937$.

[7] T. Kusano and $H$. Onose, "Oscillations of functional differential equations with retarded argument", $J$. Differential Equations 15 $(1974), 269-277$. 
[8] G. Ladas, V. Lakshmikantham, and J.S. Papadakis, "Oscillations of higher-order retarded differential equations generated by the retarded argument", Delay and functional differential equations and their applications, 219-231 (Proc. Conf. Park City, Utah, 1972. Academic Press, New York, London, 1972).

[9] J.R. Ockendon and A.B. Tayler, "The dynamics of a current collection system for an electric locomotive", Proc. Roy. Soc. London A 322 (1971), $447-468$.

Mathematical Institute,

University of Oxford,

St Giles, 